\title{
Clinical value of magnetic resonance imaging in identifying multiple cerebral gliomas from primary central nervous system lymphoma
}

\author{
YUSHAN CHEN and ALAI ZHAN \\ Department of Radiology, Zhangzhou Affiliated Hospital of Fujian Medical University, \\ Zhangzhou, Fujian 363000, P.R. China
}

Received December 4, 2018; Accepted April 12, 2019

DOI: $10.3892 / \mathrm{ol} .2019 .10352$

\begin{abstract}
Clinical value of magnetic resonance imaging (MRI) in identifying and diagnosing multiple cerebral glioma (MCG) from primary central nervous system lymphoma (PCNSL) was evaluated. A total of 21 patients with MCG diagnosed clinically and pathologically in Zhangzhou Municipal Hospital from March 2016 to April 2017 were selected as group A, and 30 patients with PCNSL diagnosed in Zhangzhou Affiliated Hospital of Fujian Medical University during the same period as group B. Plain MRI, enhanced MRI and diffusion weighted imaging (DWI) were performed in all patients, the apparent diffusion coefficient (ADC) value of lesions was measured, and the diagnostic efficacy of ADC for MCG and PCNSL was evaluated by receiver operating characteristic (ROC) curve. The incidence of hippocampus lesions, patchy and cystic lesions, and the heterogeneous signal of plain scan in group A was significantly higher than that in group $\mathrm{B}(\mathrm{P}<0.05)$, and the incidence of basal ganglia lesions was significantly lower than that in group $\mathrm{B}(\mathrm{P}<0.05)$. Mass lesions in group A were significantly less than those in group $\mathrm{B}(\mathrm{P}<0.05)$. The $\mathrm{ADC}$ value of lesions in group $\mathrm{A}$ was significantly higher than that in contralateral normal white matter $(\mathrm{P}<0.05)$, the ADC value in group $\mathrm{B}$ was significantly lower than that in normal contralateral white matter $(\mathrm{P}<0.05)$, so the ADC value in group A was significantly higher than that in group $\mathrm{B}(\mathrm{P}<0.05)$. The location, lesion shape and signal characteristic of MCG and PCNSL have their own specificity; there are significant differences in DWI signal and ADC color map signal intensity of the lesions; ADC has certain diagnostic value for MCG and PCNSL; the differential diagnosis of MCG from PCNSL by MRI is of great significance.
\end{abstract}

Correspondence to: Dr Alai Zhan, Department of Radiology, Zhangzhou Affiliated Hospital of Fujian Medical University, 59 Shengli West Road, Zhangzhou, Fujian 363000, P.R. China E-mail: ceu72q@163.com; cys6899@163.com

Key words: multiple cerebral glioma, primary central nervous system lymphoma, magnetic resonance imaging, diagnosis, apparent diffusion coefficient, weighted imaging

\section{Introduction}

Glioma is a common malignant brain tumor that threatens human health and accounts for approximately $42 \%$ of all primary adult central nervous system tumors (1). Multiple cerebral glioma (MCG) is relatively rare in clinic and has a low incidence, which is less than $10 \%$ of the total number of gliomas (2). MCG can be divided into multicentric and multifocal glioma by histopathology. Multifocal glioma infiltrates adjacent parenchyma through cerebrospinal fluid (CSF), meninges and white matter fiber tracts, and a large number of tumor cell infiltration can be found in the lesion tissues (3). While multicentric glioma is a kind of independent glioma, which is far away from partial or both cerebral hemispheres. There is no relationship between the lesions under a microscope, and their histological type can be the same or different (4). The main clinical treatment of MCG is surgery, but because of the invasive growth of the tumors and no obvious boundary between the tumors and brain tissues, resection cannot be performed in most patients, except that the early MCG patients with tumors located in the appropriate part can be completely removed (5). Therefore, it is necessary to make timely diagnosis, radiochemotherapy after surgery and other combined therapy to improve the effect of clinical treatment.

Primary central nervous system lymphoma (PCNSL) is an aggressive lymphoid neoplasm that can be seen in human cranial nerves, meninges, spinal cord and eyes, with an incidence accounting for approximately $1 \%$ of all non-Hodgkin lymphoma and 2-3\% of brain tumors (6). Moreover, its incidence in patients with low and normal immunity has increased in recent years (7). Chemotherapy is the main treatment for PCNSL, it can significantly improve the overall survival rate of patients, and can even completely eliminate lesions in some patients after treatment (8).

MCG and PCNSL have the common characteristics of infiltrative growth, high cell density and multiple lesions. Therefore, there are a large overlap and some crossed cases examined with conventional magnetic resonance imaging (MRI), which makes it more difficult to differentiate them $(9,10)$. However, it is difficult to judge the invasion of surrounding tissues by tumor cells according to the edema extent of lesions and enhancement characteristics, and the 
treatment measures of the two diseases are completely different. Therefore, accurate identification before surgery is of great significance for the follow-up treatment. The purpose of this study was to investigate the diagnostic value and differential significance of MRI in MCG and PCNSL patients by observing their MRI manifestations.

\section{Patients and methods}

General clinical data. A total of 21 patients with MCG diagnosed clinically and pathologically in Zhangzhou Affiliated Hospital of Fujian Medical University (Zhangzhou, China) from March 2016 to April 2017 were selected as group A, including 12 males and 9 females, aged 32-75 years with an average age of $56.13 \pm 12.82$ years. Main clinical symptoms: 16 cases of headache, 11 of dizziness, 7 of nausea, 5 of speech disorder, 3 of limb weakness and 2 of slow reaction. Another 30 patients with PCNSL diagnosed in Zhangzhou Affiliated Hospital of Fujian Medical University during the same period were selected as group B, including 13 males and 17 females, aged 31-73 years with an average age of $57.69 \pm 11.96$ years. Main clinical symptoms: 25 cases of headache, 18 of dizziness, 11 of nausea, 5 of speech disorder, 7 of limb weakness and 5 of slow reaction.

Inclusion and exclusion criteria. Inclusion criteria were: i) patients with MCG and PCNSL confirmed by pathology and histology $(11,12)$; ii) patients with no previous history of chemotherapy, hormone therapy, radiotherapy or craniocerebral surgery; and iii) patients with normal immune function. Exclusion criteria were: i) patients in pregnancy and lactation; ii) patients with other neoplasms, severe liver and kidney dysfunction, connective tissue diseases, endocrine metabolic diseases, hematopoietic disorders, infectious diseases; and iii) patients with mental illness or a family history of mental illness.

This study was approved by the Ethics Committee of Zhangzhou Affiliated Hospital of Fujian Medical University. Patients who participated in this research had complete clinical data. The signed informed consents were obtained from the patients or the guardians.

Instruments and testing methods. Ingenia 3.0T MR scanner (Philips Healthcare, Amsterdam, Holland). Patients received plain MRI, enhanced MRI and diffusion weighted imaging (DWI) in supine position, with the middle of the head up, well fixed, bilateral zygomorphic and the head coil being standard. Plain scan parameters: Axial, sagittal T1 weighted imaging (T1WI): TR/TE $=272 \mathrm{msec} / 2.3 \mathrm{msec}$; T2 weighted imaging (T2WI): TR/TE $=3,000 \mathrm{msec} / 80 \mathrm{msec}$; FLAIR sequences: $\mathrm{TR} / \mathrm{TE}=4,800 \mathrm{msec} / 320 \mathrm{msec}$; SE-EPI-DWI: $\mathrm{TR} / \mathrm{TE}=2,815 \mathrm{msec} / 82 \mathrm{msec}$, section thickness $=5 \mathrm{~mm}$, interval gap $=1 \mathrm{~mm}$. Enhanced MRI scan: the contrast agent was Gd-DTPA (Jinan Hongfangde Medical Technology Co., Ltd., Jinan, China) at a dose of $0.2 \mathrm{mmol} / \mathrm{kg}$, intravenous injection rate of 3-3.0 ml/sec for 3-5 sec. Axial, coronal, sagittal enhanced scan, T1WI: TR/TE $=400 \mathrm{msec} / 20 \mathrm{msec}$. SE-EPI was used for DWI, sequence: TR/TE $=5,000 \mathrm{msec} / 65 \mathrm{msec}$, 8 times acquisition, b value $=0$ and $1,000 \mathrm{sec} / \mathrm{mm}^{2}$. The matrix was $205 \times 256$, the field of vision (FOV) was $38 \times 38 \mathrm{~cm}$, the section thickness was $5.0 \mathrm{~mm}$ and the interval gap was $1.0 \mathrm{~mm}$. The maximum diameter level of the lesion was selected and the corresponding apparent diffusion coefficient (ADC) was measured in the target area where the lesion was significantly enhanced. The ADC value of the corresponding normal white matter area was measured as a reference. Measurements were repeated 3 times and the mean values were obtained.

Statistical analysis. SPSS18.0 (SPSS, Inc., Chicago, IL, USA) was used to carry out statistical analysis. The counting data were expressed as $\mathrm{n}(\%)$, and the measurement data were expressed as mean \pm standard deviation. Chi-square test was used to compare counting data among groups, t-test was used to compare measurement data among groups. The diagnostic efficacy of ADC on MCG and PCNSL was evaluated by the receiver operating characteristic (ROC) curve. $\mathrm{P}<0.05$ was considered to indicate a statistically significant difference.

\section{Results}

General data in the two groups. There was no statistically significant difference between groups A and B in general clinic data of sex, age, body mass index (BMI), smoking history, drinking history, clinical manifestations, blood glucose (Glu), alanine aminotransferase (ALT), aspartate aminotransferase (AST), carcinoembryonic antigen (CEA), lactate dehydrogenase (LDH), carbohydrate antigen 125 (CA125) or $\beta 2$-microglobulin ( $\beta 2-\mathrm{MG})(\mathrm{P}>0.05$; Table I).

Location and number of lesions in groups $A$ and B. A total of 62 lesions were detected in group A, including 13 cases in hippocampus (16 lesions), 10 cases in parietal and frontal lobe (21 lesions), 7 cases in insular and temporal lobe (9 lesions), 4 cases in thalamus ( 7 lesions), 2 cases in corpus callosum (6 lesions), 1 case in occipital lobe (1 lesion), 1 case in cerebellum (2 lesions). A total of 76 lesions were detected in group B, including 1 case in hippocampus (2 lesions), 10 cases in parietal and frontal lobe (25 lesions), 2 cases in insular and temporal lobe (5 lesions), 7 cases in thalamus (13 lesions), 3 cases in corpus callosum (7 lesions), 1 case in occipital lobe ( 3 lesions), 8 cases in basal ganglia (16 lesions) and 2 cases in cerebellum (5 lesions). The incidence of hippocampus lesions in group A was significantly higher than that in group B $(\mathrm{P}<0.05)$, and the incidence of basal ganglia lesions was significantly lower than that in group $\mathrm{B}(\mathrm{P}<0.05$; Table II).

Characteristics of plain MRI signal in groups $A$ and $B$. In group A (62 lesions), there were 32 mass lesions, 22 patchy lesions and 8 cystic lesions. A total of 35 lesions showed homogeneous signal, with low or equal T1WI signal, high or slightly high T2WI signal and 25 lesions showed heterogeneous signal, with low T1WI signal and high T2WI signal. In group B, there were 65 mass lesions and 11 patchy lesions. The signal of 62 lesions was homogeneous, 14 lesions were heterogeneous, with low or equal T1WI signal and equal or slightly high T2WI signal. Mass lesions in group A were significantly less than those in group $\mathrm{B}(\mathrm{P}<0.05)$, and the patchy and cystic lesions were significantly higher than those in group $\mathrm{B}(\mathrm{P}<0.05)$. The heterogeneous signal of plain scan in group A was significantly higher than that in group $\mathrm{B}(\mathrm{P}<0.05$; Table III). 
Table I. General data in groups A and B [n (\%)/(mean \pm SD)].

\begin{tabular}{|c|c|c|c|c|}
\hline Items & Group A $(n=21)$ & Group B $(n=30)$ & $\mathrm{t} / \chi^{2}$ & P-value \\
\hline Sex & & & 0.943 & 0.332 \\
\hline Female & $12(57.14)$ & $13(43.33)$ & & \\
\hline Male & $9(42.86)$ & $17(56.67)$ & & \\
\hline Age (years) & $56.13 \pm 12.82$ & $57.69 \pm 11.96$ & 0.445 & 0.658 \\
\hline BMI $\left(\mathrm{kg} / \mathrm{m}^{2}\right)$ & $18.23 \pm 4.16$ & $19.54 \pm 3.08$ & 1.293 & 0.202 \\
\hline Smoking history & & & 0.264 & 0.607 \\
\hline Yes & $7(33.33)$ & $8(26.67)$ & & \\
\hline No & $14(66.67)$ & $22(73.33)$ & & \\
\hline Drinking history & & & 0.199 & 0.656 \\
\hline Yes & $9(42.86)$ & $11(36.67)$ & & \\
\hline No & $12(57.14)$ & $19(63.33)$ & & \\
\hline Clinical manifestation & & & 0.148 & 0.706 \\
\hline Headache & $16(76.19)$ & $25(83.33)$ & & \\
\hline Dizziness & $11(52.38)$ & $18(60.00)$ & & \\
\hline Nausea & 7 (33.33) & $11(36.67)$ & & \\
\hline Speech disorder & $5(23.81)$ & $5(16.67)$ & & \\
\hline Limb weakness & $3(14.29)$ & $7(23.33)$ & & \\
\hline Slow reaction & $2(9.52)$ & $5(16.67)$ & & \\
\hline Glu (mmol/l) & $6.01 \pm 0.49$ & $5.93 \pm 0.28$ & 0.740 & 0.463 \\
\hline $\operatorname{ALT}(\mathrm{U} / \mathrm{l})$ & $25.63 \pm 8.48$ & $23.58 \pm 9.69$ & 0.782 & 0.438 \\
\hline AST (U/l) & $18.03 \pm 6.57$ & $19.25 \pm 7.01$ & 0.628 & 0.533 \\
\hline CEA (mg/l) & $7.41 \pm 3.56$ & $9.13 \pm 3.18$ & 1.810 & 0.077 \\
\hline LDH (U/l) & $301.52 \pm 56.38$ & $298.84 \pm 57.31$ & 0.165 & 0.869 \\
\hline CA125 (U/ml) & $28.59 \pm 8.69$ & $29.56 \pm 10.85$ & 0.340 & 0.735 \\
\hline$\beta 2-\mathrm{MG}(\mu \mathrm{g} / \mathrm{ml})$ & $3.82 \pm 1.86$ & $2.94 \pm 1.45$ & 1.898 & 0.064 \\
\hline
\end{tabular}

Glu, blood glucose; ALT, alanine aminotransferase; AST, aspartate aminotransferase; LDH, lactate dehydrogenase; CA125, carbohydrate antigen 125; $\beta 2-\mathrm{MG}, \beta 2$-microglobulin; CEA, carcinoembryonic antigen; SD, standard deviation.

Table II. Comparison of location and number of lesions between groups A and B [n (\%)].

\begin{tabular}{|c|c|c|c|c|}
\hline Location & Group A $(n=62)$ & Group B $(n=76)$ & $\chi^{2}$ & P-value \\
\hline Hippocampus & $16(25.81)$ & $2(2.63)$ & 16.170 & $<0.001$ \\
\hline Parietal lobe & $11(17.74)$ & $17(22.37)$ & 0.452 & 0.672 \\
\hline Frontal lobe & $10(16.13)$ & $8(10.53)$ & 0.945 & 0.331 \\
\hline Insular lobe & $4(6.45)$ & $2(2.63)$ & 1.198 & 0.274 \\
\hline Temporal lobe & $5(8.06)$ & $3(3.95)$ & 1.060 & 0.303 \\
\hline Thalamus & $7(11.29)$ & $13(17.11)$ & 0.932 & 0.334 \\
\hline Corpus callosum & $6(9.68)$ & $7(9.21)$ & 0.008 & 0.926 \\
\hline Occipital lobe & $1(1.61)$ & $3(3.95)$ & 0.661 & 0.416 \\
\hline Basal ganglia & $0(0.00)$ & $16(21.05)$ & 14.760 & $<0.001$ \\
\hline Cerebellum & $2(3.23)$ & $5(6.58)$ & 0.797 & 0.371 \\
\hline
\end{tabular}

Features of enhanced MRI in groups $A$ and $B$. Enhanced MRI scan in groups A and B showed that, in group A, 40 of 62 lesions were shaped as wreaths or rings, 12 lesions showed patchy or mass enhancements, and 10 lesions had no significant change. The intensity of enhancement was inconsistent in 17 cases and consistent in 4 cases. In group B, 65 of 72 lesions were homogeneous masses and nodules with obvious enhancements, and 42 of them showed sharp angle signs or fist clenching signs. There were 9 lesions with irregular or annular enhancements and 2 lesions with no obvious enhancement. 
Table III. Characteristics of plain MRI signal in groups A and B [n (\%)].

\begin{tabular}{lcrr}
\hline Characteristics of plain MRI signal & Group A (n=62) & Group B (n=76) & $\chi^{2}$ \\
\hline Lesion shape & & & P-value \\
Mass & $32(51.61)$ & $65(85.53)$ & 18.800 \\
Patchy & $22(35.48)$ & $11(14.47)$ & 8.284 \\
Cystic & $8(12.90)$ & $0(0.00)$ & 0.001 \\
Characteristics of plain scan signal & & & 0.001 \\
Homogeneous & $35(56.45)$ & $62(81.58)$ & 0.410 \\
Heterogeneous & $27(43.55)$ & $14(18.42)$ & 0.940 \\
\hline
\end{tabular}

MRI, magnetic resonance imaging.

Table IV. Diagnostic value of ADC for MCG and PCNSL.

\begin{tabular}{|c|c|c|c|c|c|c|}
\hline Diagnostic index & AUC & $95 \% \mathrm{CI}$ & $\begin{array}{l}\text { Standard } \\
\text { error }\end{array}$ & $\begin{array}{l}\text { Cut-off value } \\
\left(\mathrm{x} 10^{-3} \mathrm{~mm}^{2} / \mathrm{sec}\right)\end{array}$ & $\begin{array}{c}\text { Sensitivity } \\
(\%)\end{array}$ & $\begin{array}{l}\text { Specificity } \\
(\%)\end{array}$ \\
\hline ADC value for diagnosing $\mathrm{MCG}$ & 0.883 & $0.790-0.969$ & 0.046 & 1.094 & 85.71 & 80.00 \\
\hline ADC value for diagnosing PCNSL & 0.918 & $0.833-0.973$ & 0.044 & 0.774 & 83.33 & 86.67 \\
\hline
\end{tabular}

ADC, apparent diffusion coefficient; MCG, multiple cerebral glioma; PCNSL, primary central nervous system lymphoma.

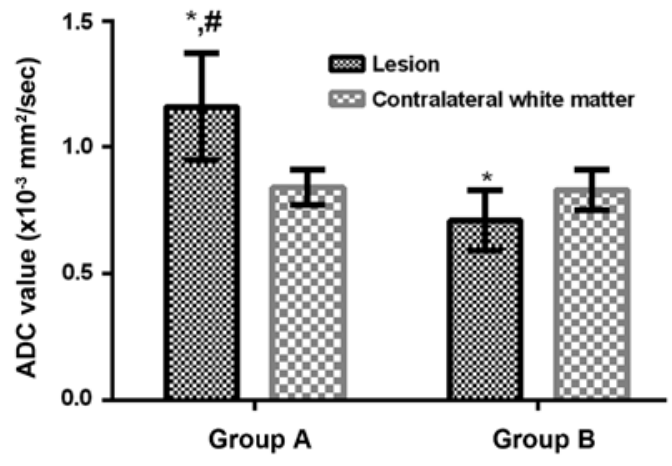

Figure 1. Comparison of ADC value of lesions and normal white matter between groups $\mathrm{A}$ and $\mathrm{B}$. The ADC value of the lesions in group A was significantly higher than that in the contralateral normal white matter $(\mathrm{P}<0.05)$. The $\mathrm{ADC}$ value in group $\mathrm{B}$ was significantly lower than that in contralateral normal white matter $(\mathrm{P}<0.05)$. The $\mathrm{ADC}$ value in group $\mathrm{A}$ was significantly higher than that in group $\mathrm{B}(\mathrm{P}<0.05)$. Compared with contralateral white matter, ${ }^{*} \mathrm{P}<0.05$; compared with the ADC value in group $\mathrm{B},{ }^{\sharp} \mathrm{P}<0.05$. ADC, apparent diffusion coefficient.

DWI findings in groups $A$ and B. In group A, 27 of 62 lesions showed equal, slightly high or high signals in DWI and slightly high or high signals in ADC color map, 25 lesions showed equal signals in DWI and high signals in ADC color map and 10 lesions showed low signals in DWI and slightly high in ADC color map. In group B, 63 of 76 lesions showed slightly high or high signals in DWI and slightly low or low signals in ADC color map and 13 lesions showed equal signals in DWI and ADC color map. The ADC value of the lesions in group A was significantly higher than that in the contralateral normal white matter $(\mathrm{P}<0.05)$, the ADC value in group B was significantly lower than that in contralateral

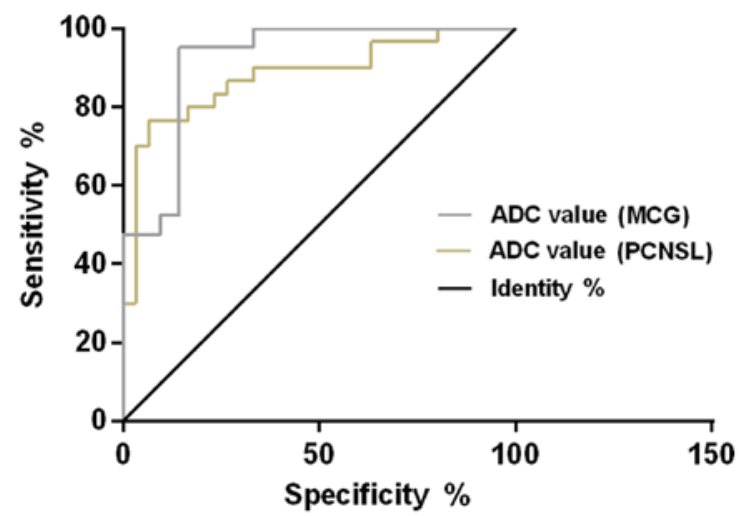

Figure 2. ROC curve of ADC value for diagnosing MCG and PCNSL. The AUC of ADC value for diagnosing MCG was 0.883 (95\% CI: 0.790-0.969), the cut-off value was $1.094 \times 10^{-3} \mathrm{~mm}^{2} / \mathrm{sec}$, the diagnostic sensitivity was $85.71 \%$ and the specificity was $80.00 \%$. The AUC of ADC value for diagnosing PCNSL was 0.918 (95\% CI: 0.833-0.973), the cut-off value was $0.774 \times 10^{-3} \mathrm{~mm}^{2} / \mathrm{sec}$, the diagnostic sensitivity was $83.33 \%$ and the specificity was $86.67 \%$. ROC, receiver operating characteristic; ADC, apparent diffusion coefficient; MCG, multiple cerebral glioma; PCNSL, primary central nervous system lymphoma.

normal white matter $(\mathrm{P}<0.05)$, so the $\mathrm{ADC}$ value in group $\mathrm{A}$ was significantly higher than that in group $\mathrm{B}(\mathrm{P}<0.05$; Fig. 1$)$. The ROC curve of ADC value for diagnosing $M C G$ and PCNSL showed that, the AUC of ADC value for diagnosing MCG was 0.883 (95\% CI: 0.790-0.969), the cut-off value was $1.094 \times 10^{-3} \mathrm{~mm}^{2} / \mathrm{sec}$, the diagnostic sensitivity was $85.71 \%$ and the specificity was $80.00 \%$; the AUC of ADC value for diagnosing PCNSL was 0.918 (95\% CI: 0.833-0.973), the cut-off value was $0.774 \times 10^{-3} \mathrm{~mm}^{2} / \mathrm{sec}$, the diagnostic sensitivity was $83.33 \%$ and the specificity was $86.67 \%$ (Table IV and Fig. 2). 


\section{Discussion}

MCG has the characteristics of rapid development, different degree of lesion differentiation, short course of disease and its main clinical treatment is surgical resection, followed by radiochemotherapy for comprehensive treatment, whereas PCNSL is sensitive to radiochemotherapy (13). MCG and PCNSL are completely different in clinically preferred treatment options. Therefore, the early preoperative differential diagnosis of them is of great significance to the prognosis of patients.

The pathogenesis of MCG has not been clarified, and its lesions can occur simultaneously, or at intervals of months or even years. It was previously believed that MCG may be derived from the the differentiation of primordial cells displaced during the development of the central nervous system, or the multiple lesions formed by the local metastasis of gliomas through CSF, blood and nerve fibers $(14,15)$. Hartmann et al (16) showed that MCG mostly occurred in the hippocampus, accounting for $70 \%$, followed by temporal lobe, insular lobe, corpus callosum, parietal lobe, frontal lobe and thalamus. Because most of the PCNSL cells originate from mononuclear phagocytes in the perivascular, and the space near the surface of internal brain and the paraventricular vessels are more obvious, most tumors occur in the deep brain tissue near the midline. The invasive and infiltrative growth of tumor cells is conducted from the center to the periphery, so the tumor is more common in the frontal and parietal lobe, corpus callosum, basal ganglia and periventricular region (17). In this study, MCG tumors mostly occurred in hippocampus, frontal lobe, insular lobe, temporal lobe, thalamus and corpus callosum, PCNSL tumors mostly occurred in frontal and parietal lobe, thalamus, corpus callosum, and basal ganglia. The incidence of hippocampus lesions in group A was significantly higher than that in group B, and the incidence of basal ganglia lesions was significantly lower than that in group B. Therefore, there is a certain difference between MCG and PCNSL in the location of tumorigenesis. Mass lesions in group A were significantly less than those in group B, and the patchy and cystic lesions were significantly higher than those in group B. The heterogeneous signal of plain scan in group A was significantly higher than that in group $\mathrm{B}$. These results indicated that the shape and signal uniformity of MCG are significantly different from those of PCNSL. Enhanced MRI showed that the enhancement characteristic of MCG was mostly shaped as wreaths or rings, which may be caused by the rapid development of MCG, prone to cystic degeneration, necrosis and hemorrhage, rich blood volume of tumors, more neovascularization, and obvious proliferation of vascular smooth muscle cells and vascular endothelial cells in the lesions (18). The enhancement of PCNSL was mainly characterized by sharp angle sign or fist clenching sign, which may be that PCNSL cells show infiltration-like pathological changes which damage vascular endothelium and the barrier between blood and CSF. And when performing an enhanced scan, the contrast agent can infiltrate into the extracellular space of the tissue through the blood vessels to obviously enhance the the lesion tissues. Moreover, lymphoma grows fast and infiltrates rapidly, and tumor cells can infiltrate along the surrounding brain parenchyma (19).
The DWI signal intensity of different gliomas is different because high grade gliomas are characterized by high cell density and high proliferation, while low grade gliomas by slow invasive growth (20). PCNSL cells are numerous and dense, with less extracellular space and higher intracellular water viscosity, which can limit the diffusion of water molecules in tumor tissues. Therefore, the lesions show a high signal in DWI and a decreased ADC value (21). In this study, the PCNSL patients showed slightly high or high signal intensity in DWI, and slightly low or low signal intensity in ADC color map, the ADC value of lesions in group B was significantly lower than that in contralateral normal white matter. The intensity of DWI signal in MCG patients was different, the ADC value of the lesion in group A was significantly higher than that in the contralateral normal white matter, and the contradictory phenomenon of high DWI signal and increased ADC value appeared. Guo et al (22) suggested that it was due to the penetrating effect of $\mathrm{T} 2$, not the limitation of tissue diffusion. ADC value can reflect the diffusion of tumor tissue by eliminating the anisotropy, diffusion sensitivity gradient and penetration effect of T2 (21).

Recent studies have shown that MRI findings and ADC values are valuable in the diagnosis of PCNSL and glioma $(23,24)$. The results of this study showed that the ADC value of lesions in group A was significantly higher than that in group B, indicating that the diffusion limitation of tumor lesion in PCNSL is higher than that in MCG. The ROC curve of ADC value for diagnosis of MCG and PCNSL found that, for MCG, cut-off value was $1.094 \times 10^{-3} \mathrm{~mm}^{2} / \mathrm{sec}$, the diagnostic sensitivity was $85.71 \%$ and the specificity was $80.00 \%$; while for PCNSL, the cut-off value was $0.774 \times 10^{-3} \mathrm{~mm}^{2} / \mathrm{sec}$, the diagnostic sensitivity was $83.33 \%$ and the specificity was $86.67 \%$, suggesting that ADC value has certain diagnostic value for MCG and PCNSL. This is similar to the study of Ahn et al (25), which suggested that ADC value was helpful in differentiating and diagnosing central nervous system lymphoma and glioblastoma. Therefore, ADC value may become an important auxiliary diagnostic method for MCG and PCNSL.

The subjects in this study were screened strictly according to the exclusion criteria. There was no significant difference in sex, age, BMI, smoking history, drinking history, clinical manifestation, Glu, ALT, AST, CEA, LDH, CA125 or $\beta 2-\mathrm{MG}$ between groups $\mathrm{A}$ and $\mathrm{B}$, which ensures the reliability of the investigation. However, the gold criteria for the diagnosis of MCG and PCNSL need to be histopathologically diagnosed. There were some limitations in this study, MCG and PCNSL patients were not classified by pathology, and the MRI signs were not known. In future research, pathological histology should be combined to explore MRI signs of MCG and PCNSL with different types.

In conclusion, the location, lesion shape and signal characteristics of MCG and PCNSL have their own specificity; there are significant differences in DWI signal and ADC color map signal intensity of the lesions; ADC value has certain diagnostic value for MCG and PCNSL; the differential diagnosis of MCG from PCNSL by MRI is of great significance.

\section{Acknowledgements}

Not applicable. 


\section{Funding}

No funding was received.

\section{Availability of data and materials}

The datasets used and/or analyzed during the present study are available from the corresponding author on reasonable request.

\section{Authors' contributions}

YC drafted, wrote the manuscript and analyzed MRI results. AZ collected and analyzed general data of patients. Both authors read and approved the final manuscript.

\section{Ethics approval and consent to participate}

This study was approved by the Ethics Committee of Zhangzhou Affiliated Hospital of Fujian Medical University (Zhangzhou, China). Patients who participated in this research had complete clinical data. The signed informed consents were obtained from the patients or the guardians.

\section{Patient consent for publication}

Not applicable.

\section{Competing interests}

The authors declare that they have no competing interests.

\section{References}

1. Salvati M, Caroli E, Orlando ER, Frati A, Artizzu S and Ferrante L: Multicentric glioma: our experience in 25 patients and critical review of the literature. Neurosurg Rev 26: 275-279, 2003.

2. Yin L and Zhang L: Correlation between MRI findings and histological diagnosis of brainstem glioma. Can J Neurol Sci 40: 348-354, 2013

3. Nakhl F, Chang EM, Shiau JS, Alastra A, Wrzolek M, Odaimi M, Raden $\mathrm{M}$ and Juliano JE: A patient with multiple synchronous gliomas of distinctly different grades and correlative radiographic findings. Surg Neurol Int 1: 48, 2010.

4. di Russo P, Perrini P, Pasqualetti F, Meola A and Vannozzi R: Management and outcome of high-grade multicentric gliomas: a contemporary single-institution series and review of the literature. Acta Neurochir (Wien) 155: 2245-2251, 2013.

5. Nassiri M, Byrne GE, Whitcomb CC and Byrnes JJ: Synchronous null-cell anaplastic large cell lymphoma and multiple myeloma. Ann Hematol 88: 923-925, 2009.

6. Fine HA and Mayer RJ: Primary central nervous system lymphoma. Ann Intern Med 119: 1093-1104, 1993.

7. Viaccoz A, Ducray F, Tholance Y, Barcelos GK. Thomas-Maisonneuve L, Ghesquières H, Meyronet D, Quadrio I Cartalat-Carel S, Louis-Tisserand G, et al: CSF neopterin level as a diagnostic marker in primary central nervous system lymphoma. Neuro Oncol 17: 1497-1503, 2015.

8. Hoang-Xuan K, Bessell E, Bromberg J, Hottinger AF, Preusser M, Rudà R, Schlegel U, Siegal T, Soussain C, Abacioglu U, et al; European Association for Neuro-Oncology Task Force on Primary CNS Lymphoma: Diagnosis and treatment of primary CNS lymphoma in immunocompetent patients: guidelines from the European Association for Neuro-Oncology. Lancet Oncol 16 : e322-e332, 2015.
9. Wang K, Zhao X, Chen Q, Fan D, Qiao Z, Lin S, Jiang T, Dai J and Ai L: A new diagnostic marker for differentiating multicentric gliomas from multiple intracranial diffuse large B-cell lymphomas on 18F-FDG PET images. Medicine (Baltimore) 96: e7756, 2017.

10. Nagashima H, Sasayama T, Tanaka K, Kyotani K, Sato N, Maeyama M, Kohta M, Sakata J, Yamamoto Y, Hosoda K, et al: Myo-inositol concentration in MR spectroscopy for differentiating high grade glioma from primary central nervous system lymphoma. J Neurooncol 136: 317-326, 2018.

11. Huang J, Yu J and Peng Y: Association between dynamic contrast enhanced MRI imaging features and WHO histopathological grade in patients with invasive ductal breast cancer. Oncol Lett 11: 3522-3526, 2016.

12. Sugita Y, Muta H, Ohshima K, Morioka M, Tsukamoto Y, Takahashi $\mathrm{H}$ and Kakita A: Primary central nervous system lymphomas and related diseases: Pathological characteristics and discussion of the differential diagnosis. Neuropathology 36: 313-324, 2016.

13. Ferreri AJ, Reni M, Foppoli M, Martelli M, Pangalis GA, Frezzato M, Cabras MG, Fabbri A, Corazzelli G, Ilariucci F, et al; International Extranodal Lymphoma Study Group (IELSG): High-dose cytarabine plus high-dose methotrexate versus high-dose methotrexate alone in patients with primary CNS lymphoma: a randomised phase 2 trial. Lancet 374: 1512-1520, 2009.

14. Lee JS, Jung TY, Lee KH and Kim SK: Primary central nervous system vasculitis mimicking a cortical brain tumor: a case report. Brain Tumor Res Treat 5: 30-33, 2017.

15. Weller RO, Galea I, Carare RO and Minagar A: Pathophysiology of the lymphatic drainage of the central nervous system: implications for pathogenesis and therapy of multiple sclerosis. Pathophysiology 17: 295-306, 2010.

16. Hartmann M, Heiland S, Harting I, Tronnier VM, Sommer C, Ludwig R and Sartor K: Distinguishing of primary cerebral lymphoma from high-grade glioma with perfusion-weighted magnetic resonance imaging. Neurosci Lett 338: 119-122, 2003.

17. Haldorsen IS, Espeland A and Larsson EM: Central nervous system lymphoma: Characteristic findings on traditional and advanced imaging. AJNR Am J Neuroradiol 32: 984-992, 2011.

18. Rojiani AM and Dorovini-Zis K: Glomeruloid vascular structures in glioblastoma multiforme: an immunohistochemical and ultrastructural study. J Neurosurg 85: 1078-1084, 1996.

19. Fraser E, Gruenberg K and Rubenstein JL: New approaches in primary central nervous system lymphoma. Chin Clin Oncol 4: $11,2015$.

20. Kono K, Inoue Y, Nakayama K, Shakudo M, Morino M, Ohata K, Wakasa K and Yamada R: The role of diffusion-weighted imaging in patients with brain tumors. AJNR Am J Neuroradiol 22: 1081-1088, 2001

21. Koubska E, Weichet J and Malikova H: Central nervous system lymphoma: a morphological MRI study. Neuro Endocrinol Lett 37: 318-324, 2016.

22. Guo AC, Cummings TJ, Dash RC and Provenzale JM: Lymphomas and high-grade astrocytomas: comparison of water diffusibility and histologic characteristics. Radiology 224: 177-183, 2002.

23. Choi YS, Lee HJ, Ahn SS, Chang JH, Kang SG, Kim EH, Kim SH and Lee SK: Primary central nervous system lymphoma and atypical glioblastoma: differentiation using the initial area under the curve derived from dynamic contrast-enhanced MR and the apparent diffusion coefficient. Eur Radiol 27: 1344-1351, 2017.

24. Gadda D, Mazzoni LN, Pasquini L, Busoni S, Simonelli P and Giordano GP: Relationship between apparent diffusion coefficients and MR spectroscopy findings in high-grade gliomas. J Neuroimaging 27: 128-134, 2017.

25. Ahn SJ, Shin HJ, Chang JH and Lee SK: Differentiation between primary cerebral lymphoma and glioblastoma using the apparent diffusion coefficient: Comparison of three different ROI methods. PLoS One 9: e112948, 2014.

This work is licensed under a Creative Commons Attribution-NonCommercial-NoDerivatives 4.0 International (CC BY-NC-ND 4.0) License. 